\title{
Hedgehog and retinoid signaling alters multiple myeloma microenvironment and generates bortezomib resistance
}

\author{
Salvador Alonso, Daniela Hernandez, Yu-ting Chang, Christian B. Gocke, Megan McCray, Ravi Varadhan, William H. Matsui, \\ Richard J. Jones, and Gabriel Ghiaur
}

Sidney Kimmel Comprehensive Cancer Center, Department of Oncology, Johns Hopkins University, Baltimore, Maryland, USA.

\begin{abstract}
Interactions between multiple myeloma (MM) cells and the BM microenvironment play a critical role in bortezomib (BTZ) resistance. However, the mechanisms involved in these interactions are not completely understood. We previously showed that expression of CYP26 in BM stromal cells maintains a retinoic acid-low (RA-low) microenvironment that prevents the differentiation of normal and malignant hematopoietic cells. Since a low secretory B cell phenotype is associated with BTZ resistance in $\mathrm{MM}$ and retinoid signaling promotes plasma cell differentiation and Ig production, we investigated whether stromal expression of the cytochrome P450 monooxygenase CYP26 modulates BTZ sensitivity in the BM niche. CYP26mediated inactivation of RA within the BM microenvironment prevented plasma cell differentiation and promoted a B celllike, BTZ-resistant phenotype in human MM cells that were cocultured on BM stroma. Moreover, paracrine Hedgehog secretion by MM cells upregulated stromal CYP26 and further reinforced a protective microenvironment. These results suggest that crosstalk between Hedgehog and retinoid signaling modulates BTZ sensitivity in the BM niche. Targeting these pathological interactions holds promise for eliminating minimal residual disease in MM.
\end{abstract}

\section{Introduction}

Multiple myeloma (MM) is characterized by the proliferation of malignant plasma cells (PCs) within the BM and their production of monoclonal Ig. Novel therapies, including proteasome inhibitors, have significantly extended the survival of patients with MM but have failed to achieve a cure. Increasing evidence demonstrates that interactions with the BM microenvironment play a critical role in the survival of MM cells during chemotherapy (1-3). However, the mechanisms mediating this $\mathrm{BM}$ niche-dependent chemoprotection are incompletely understood and remain a critical area of research.

We and others have demonstrated the existence of MM cells that resemble mature B cells and are resistant to bortezomib (BTZ) $(4,5)$. Like their normal B cell counterparts, these CD138- MM cells are capable of clonogenic growth and differentiation into CD138 ${ }^{+}$PCs; moreover, these cells are enriched during minimal residual disease (MRD) (4), suggesting a critical role in disease relapse. Differential BTZ sensitivity of $\mathrm{CD} 138^{+}$and $\mathrm{CD} 138^{-} \mathrm{MM}$ cells may be explained by their secretory activity (6). As a result of their abundant Ig production, CD $138^{+}$PCs are highly dependent on an intact proteasome pathway to degrade improperly folded proteins. Conditions that disrupt protein degradation by the proteasome activate a cellular stress pathway known as the unfolded protein response (UPR), which counteracts ER stress by decreasing protein synthesis and promoting protein degradation (7). If homeostasis cannot be reestablished, UPR activation eventually leads to apoptosis. On the other hand, CD138- MM cells exhibit limited Ig production and low ER stress and are less dependent on proteasome-mediated degradation of misfolded proteins $(4,5)$.

Conflict of interest: The authors have declared that no conflict of interest exists. Submitted: April 19, 2016; Accepted: September 15, 2016.

Reference information: / Clin Invest. 2016;126(12):4460-4468. doi:10.1172/JCI88152.
Previous studies demonstrated that BM stromal cells induce an immature drug-resistant phenotype in $\mathrm{MM}(8,9)$. We have shown that BM stroma creates a retinoic acid-low (RA-low) environment via CYP26 that prevents the differentiation of normal and malignant cells $(10,11)$. Since retinoid signaling promotes PC differentiation and Ig production $(12,13)$, we tested whether the BM niche via stromal CYP26 activity induces BTZ resistance by preventing PC differentiation. We show here that an RA-low environment induced by stromal CYP26 is responsible for maintaining a B cell-like, BTZ-resistant phenotype in MM cells. Directly inhibiting CYP26 or bypassing stromal protection via a CYP26-resistant retinoid rescues PC differentiation and BTZ sensitivity. Furthermore, we describe a bidirectional crosstalk, in which paracrine Hedgehog secreted by MM cells reinforces a protective niche via an increase in the ability of BM stroma to inactivate RA. These data indicate that modulation of RA signaling is an attractive therapeutic strategy for overcoming BTZ resistance in the MM BM microenvironment.

\section{Results}

The BM niche limits PC differentiation by modulating retinoid signaling. We and others have found that a population of MM progenitors, phenotypically similar to B cells, is intrinsically resistant to BTZ and contributes to MRD and relapse $(4,5)$. To investigate whether the BM niche plays a role in determining the phenotype of MM cells, we analyzed the mRNA expression of B cell and PC markers in MM cell lines (H929, MM1S, and U266) and MM $\mathrm{CD}_{138^{+}}$primary cells following coculture with mouse BM stroma using human-specific primers. B cell lymphoma 6 (BCL6), a transcriptional repressor that promotes self-renewal of germinal center B cells and prevents PC differentiation (14), was upregulated in the presence of BM stromal cells (Figure 1, A and B, and Sup- 

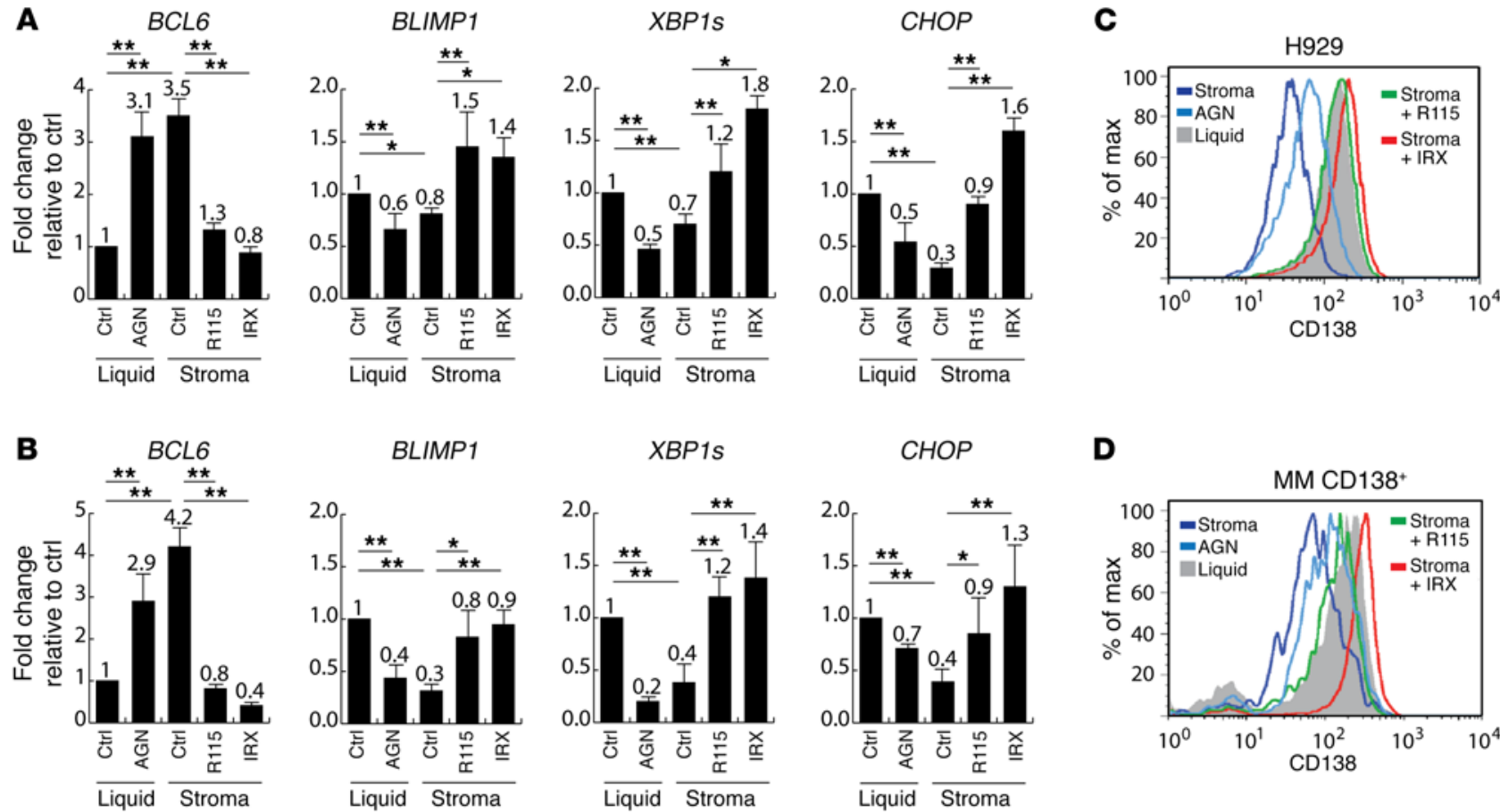

Figure 1. Expression of $\mathbf{B}$ and plasma cell markers in response to retinoid-low or -high conditions. (A and $\mathbf{B}$ ) Relative quantification of $B C L 6$ (B cell marker), BLIMP, XBP1s, and CHOP (plasma cell markers) in $\mathrm{H} 929$ cells (A) or CD138+ MM cells (B) from 3 different patient samples incubated for 5 days either in the absence of stroma (Liquid), with or without AGN (RA receptor antagonist, $1 \mu \mathrm{M}$ ), or cocultured with BM mesenchymal cells (Stroma), with or without R115 (CYP26 inhibitor, $1 \mu \mathrm{M}$ ) or IRX (CYP26-resistant retinoid, $1 \mu \mathrm{M}$ ). Expression in untreated liquid conditions was set at 1. (C and D) Flow cytometric analysis of CD138 in H929 cells (C) or primary CD138+ MM cells (D) incubated for 5 days in the absence of stroma (Liquid), with or without AGN, or in the presence of BM mesenchymal cells (Stroma), with or without R115 $(1 \mu \mathrm{M})$ or IRX $(1 \mu \mathrm{M})$. Data are representative of 3 independent experiments with similar results and represent the mean $\pm \mathrm{SEM} .{ }^{*} P \leq 0.05$ and ${ }^{* *} P \leq 0.01$, by repeated-measures 1 -way ANOVA for determination of statistical significance between groups; $P$ values were corrected for multiple comparisons using Dunnett's test. Ctrl, control; max, maximum.

plemental Figure 1; supplemental material available online with this article; doi:10.1172/JCI88152DS1). In contrast, coculture of MM cells with BM stroma decreased the mRNA expression of $\mathrm{B}$ lymphocyte-induced maturation protein 1 (BLIMP1) and spliced $\mathrm{X}$ box-binding protein 1 (XBP1s) (Figure 1, A and B, and Supplemental Figure 1), which are critical mediators of PC differentiation (14, 15). Similarly, C/EBP homologous protein (CHOP), a key component of the UPR pathway (15), was downregulated in the presence of BM stromal cells (Figure 1, A and B, and Supplemental Figure 1).

We showed that the BM niche regulates hematopoietic stem cell (HSC) differentiation by expressing the retinoid-inactivating enzyme CYP26 (10). These findings have been confirmed by others in an in vivo model (16). As shown previously, CYP26 enzymes were highly expressed in BM mesenchymal cells (10), while their expression was barely detectable in MM cells (data not shown). Since retinoid signaling promotes PC differentiation and potentiates Ig secretion $(12,13)$, we tested whether stromal CYP26 is responsible for inducing a B cell phenotype in MM cells $(8,9)$. To this end, coculture conditions were treated with the CYP26 inhibitor R115866 (R115) (10) or the CYP26-resistant RA receptor $\alpha-$ selective (RAR $\alpha$-selective) retinoid IRX5183 (IRX) (17). Incubation of stroma cocultures with either R115 or IRX restored all markers to levels comparable to those of liquid control conditions (Figure 1, A and $\mathrm{B})$. Moreover, treatment of MM cells with the pan-RAR antagonist AGN194310 (AGN) (10) mimicked the changes induced by BM stromal cells (Figure 1, A and B), limiting PC differentiation.
Expression of CD138 is a hallmark of normal PC differentiation as well MM PCs. Consistent with mRNA levels of PC markers, surface CD138 expression was markedly decreased by coculture with BM stromal cells or incubation with AGN (Figure 1, C and D). Incubation of BM stromal cell cocultures with R115 or IRX restored CD138 expression in MM cells. R115 did not significantly affect the expression of differentiation markers in liquid conditions by quantitative reverse transcription-PCR (qRT-PCR) or flow cytometry, while IRX induced comparable changes, irrespective of the presence or absence of BM stroma (data not shown). Taken together, these data suggest that retinoid signaling promotes PC differentiation of MM cells and that this process is blocked by stromal CYP26mediated metabolism of RA.

A RA-low microenvironment induces $B T Z$ resistance. To determine whether decreased retinoid signaling contributes to BTZ resistance within the $\mathrm{BM}$ niche, $\mathrm{MM}$ cell lines and $\mathrm{MM} \mathrm{CD} 138^{+}$primary cells were incubated with BM stroma for 5 days, followed by BTZ treatment. In the absence of BM stroma (liquid), MM cells were highly sensitive to BTZ (Figure 2A and Supplemental Figure 2). However, incubation with BM stroma induced BTZ resistance, which was overcome by CYP26 inhibition via R115 or by the CYP26-resistant retinoid IRX. Moreover, treatment of MM cells with the pan-RAR antagonist AGN mimicked the changes induced by BM stromal cells (Figure 2A and Supplemental Figure 2), decreasing BTZ sensitivity.

Strategies to overcome microenvironment-dependent chemoprotection have focused on mobilization of cancer cells from 
A

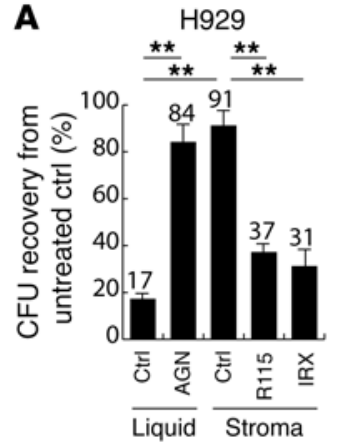

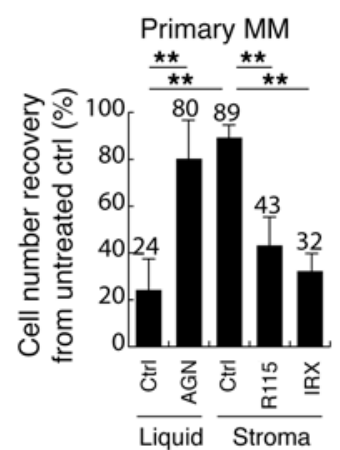

B

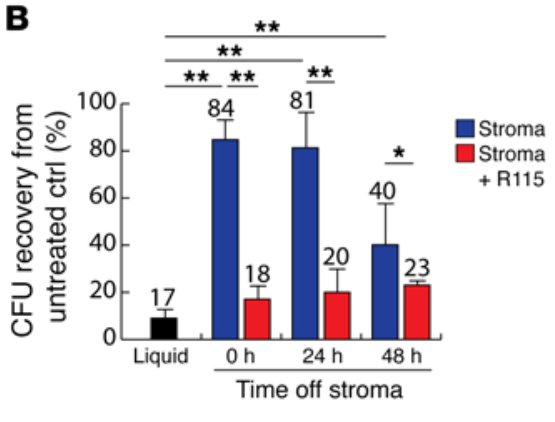

C
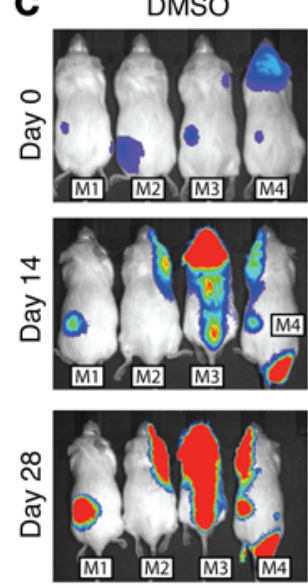

$148.2 \pm 26$
IRX

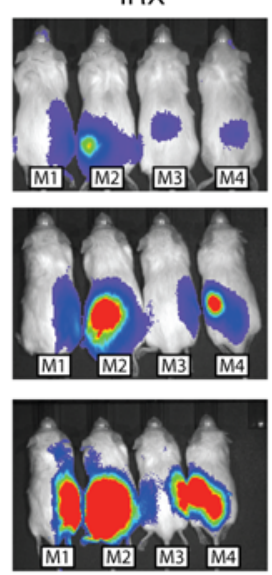

$29.3 \pm 6$

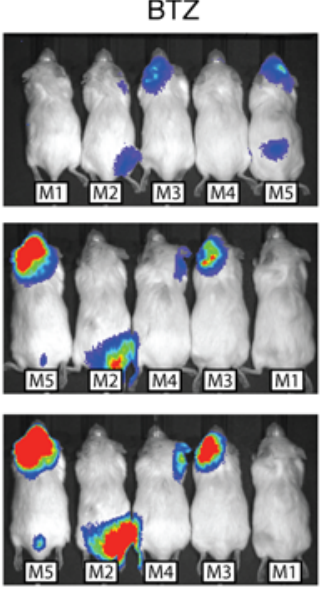

$3.2 \pm 1.8$
$\mathrm{IRX}+\mathrm{BTZ}$
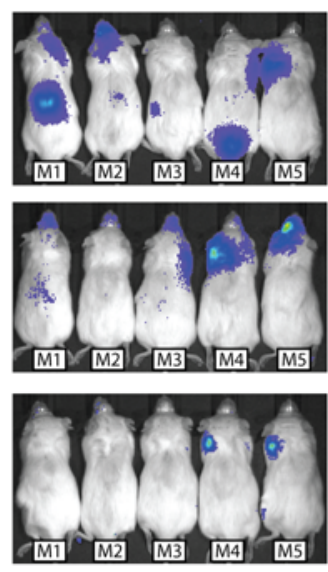

$0.3 \pm 0.1$

Bioluminescence fold change from day 0

D

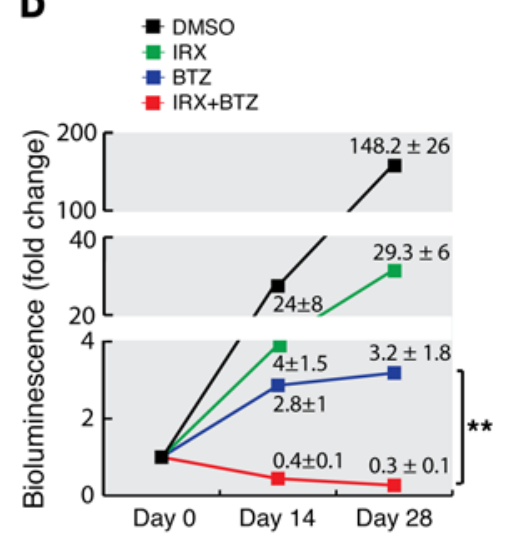

Figure 2. BTZ sensitivity of the MM cell line $\mathrm{H} 929$ and primary CD138 MM cells in various retinoid-low and -high conditions. (A) Clonogenic recovery (CFU) of $\mathrm{H} 929$ cells or cellular recovery of primary CD138+ MM cells from 3 different patient samples. MM cells were treated with BTZ (2.5 nM) for 48 hours after being incubated for 5 days either in the absence of stroma (Liquid), with or without the pan-RAR inhibitor ACN ( $1 \mu M)$, or in the presence of BM mesenchymal cells (Stroma), with or without the CYP26 inhibitor R115 $(1 \mu \mathrm{M})$ or the CYP26-resistant retinoid IRX (1 $\mu \mathrm{M})$. Clonogenic or cellular recovery was normalized to each condition in the absence of BTZ. (B) Clonogenic recovery of H929 cells treated with BTZ ( 2.5 nM). MM cells were incubated for 5 days in the absence (Liquid) or presence of BM mesenchymal cells (Stroma), with or without R115 (1 $\mu \mathrm{M})$. Following this preincubation, H929 cells were separated from BM stroma, cultured in fresh media for 0 to 48 hours, and then treated with BTZ ( $2.5 \mathrm{nM})$ for 48 hours. Clonogenic recovery was normalized to each condition in the absence of BTZ. (C) Bioluminescent images of systemic MM xenografts. Following engraftment of $\mathrm{H}^{2} 2 \mathrm{~S}$ Luc $^{+}$cells, mice were treated with IRX $(n=4)$, BTZ $(n=5)$, or a combination of both $(n=5)$ for 4 weeks. Data represent the mean \pm SEM of the fold change in bioluminescence (photons/second) from day 0 . (D) Fold change in bioluminescence from day 0 . Data represent the average of 4 to 5 independent xenografts and represent the mean \pm SEM. ${ }^{*} P \leq 0.05$ and ${ }^{* *} P \leq 0.01$, by unpaired, 2 -tailed Student's $t$ test.

the BM niche into the peripheral circulation $(18,19)$. Accordingly, clinical studies combining the CXCR4 inhibitor plerixafor with BTZ are ongoing (20). We therefore analyzed whether the change in phenotype and subsequent BTZ resistance of MM cells were lost upon separation from the BM stroma, a process that mimics mobilization. To this end, H929 cells were separated from BM mesenchymal cells following a 5-day stroma coculture, incubated in fresh media (RPMI with 10\% FBS) for 0 to 48 hours, and then treated with BTZ. Interestingly, MM cells remained partially resistant to BTZ for up to 48 hours following detachment from stroma (Figure 2B). Moreover, treatment of the coculture conditions with R115 prevented the development of a BTZ-resistant phenotype (Figure 2B). Thus, microenvironment-dependent BTZ resistance induced by the change in MM cell phenotype may not immediately be reversed by tumor mobilization.

To test whether retinoids can enhance BTZ activity in MM, we generated a systemic MM xenograft by injecting $2 \times 10^{6} \mathrm{H} 929$ luciferase ${ }^{+}\left(\mathrm{Luc}^{+}\right)$cells via the tail vein of nonobese, diabetic, severe combined immunodeficiency IL-2 receptor $\gamma$-KO (NSG) mice. The animals were randomized to receive IRX, BTZ, or a combination of both, and disease burden was followed weekly by bioluminescence imaging (Figure 2C). Mice treated with BTZ showed decreased tumor growth compared with untreated controls (Figure 2D); however, some MM cells remained resistant to BTZ, as demonstrated by the continued increase in bioluminescence. Similarly, mice treated with IRX monotherapy showed a decrease in tumor burden compared with untreated mice. Most important, IRX sensitized MM cells to BTZ, leading to a significant $(P<0.01)$ decrease in disease burden (Figure 2D). Collectively, these data suggest that an RA-low microenvironment created by stromal CYP26 induces a BTZ-resistant phenotype, which is maintained even after displacement from the BM niche.

$M M$ cells induce stromal CYP26. Recent studies have demonstrated the existence of a bi-directional crosstalk, in which not 

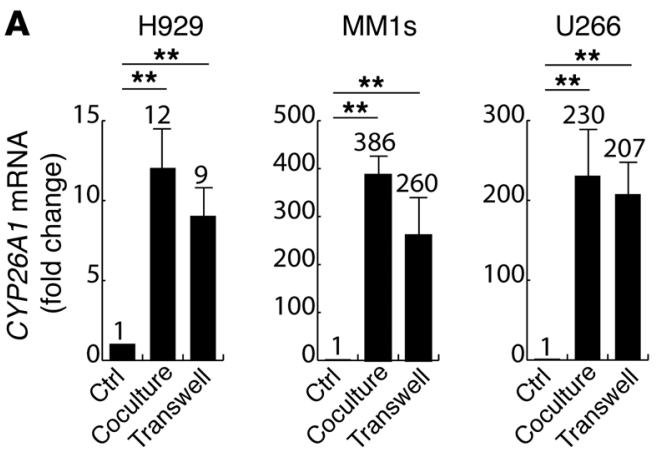

B

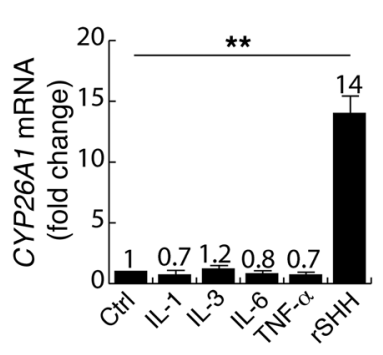

C

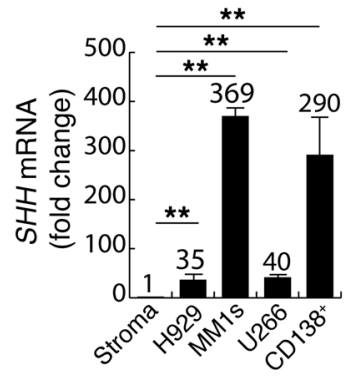

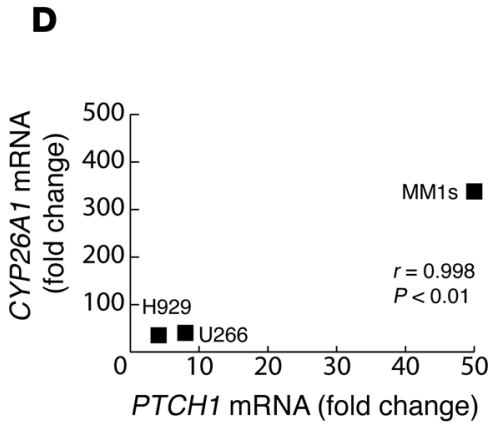

$\mathbf{E}$

H929
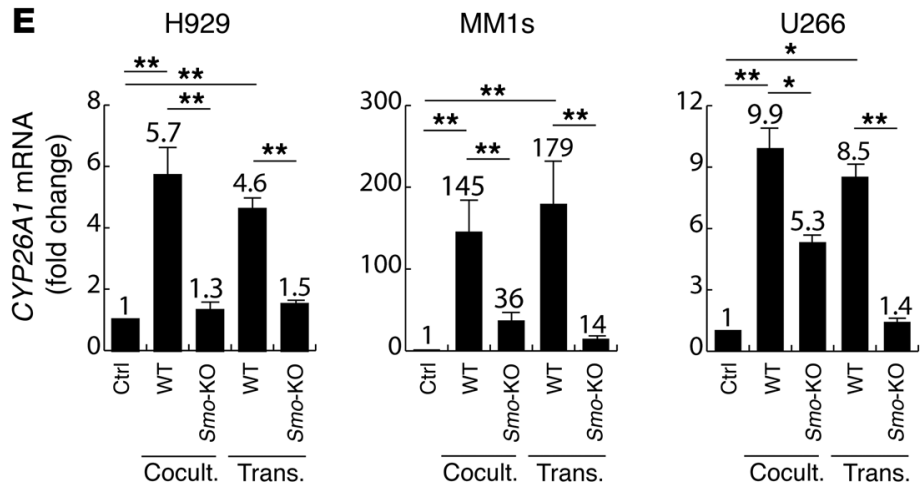

Figure 3. Effects of MM cells on the expression of CYP26A1 in BM stroma. (A) Relative quantification of CYP26A1 mRNA in human BM mesenchymal cells incubated for 24 hours either in the absence (Ctrl) or presence (Coculture or Transwell) of MM cells (H929, MM1s, U266). Expression in untreated BM stroma (Ctrl) was arbitrarily set at 1. (B) Relative quantification of CYP26A1 mRNA in human BM mesenchymal cells treated for 24 hours with soluble factors secreted by MM cells (IL-1, IL-3, IL-6, TNF- $\alpha$, and SHH). Expression in untreated BM stromal cells was set at 1. (C) Relative quantification of SHH mRNA in human BM stroma, MM cells lines (H929, MM1S, U266), and primary CD138+ MM cells from 3 different patient samples. Expression in BM stromal cells was set at 1. (D) Correlation between induction of CYP26A1 mRNA and induction of PTCH mRNA expression in human BM mesenchymal cells cocultured with the indicated MM cells. $R$ and $P$ values were calculated using Pearson's correlation coefficient. (E) Relative quantification of CYP26A1 mRNA in mouse WT or Smo-KO BM stroma incubated for 24 hours in the absence (Ctrl) or presence (Coculture or Transwell) of MM cells (H292, MM1s, U266). Expression in untreated WT or Smo-KO stroma was arbitrarily set at 1 for the respective treated conditions. Data depicted in $\mathbf{A}-\mathbf{C}$ and $\mathbf{E}$ represent the mean \pm SEM of 3 independent experiments. ${ }^{*} P \leq 0.05$ and ${ }^{* *} P \leq 0.01$, by unpaired, 2 -tailed Student's $t$ test.

only stromal cells provide a protective microenvironment, but also cancer cells actively adapt and build a reinforced niche (21-24). Thus, we investigated whether MM cells reinforce a protective microenvironment by strengthening the ability of BM stroma to inactivate retinoids. Stromal CYP26 expression was analyzed by qRT-PCR in BM mesenchymal cells following a 24-hour coculture with MM cells. The isoenzyme CYP26A1 was highly upregulated by all $3 \mathrm{MM}$ cell lines tested (Figure $3 \mathrm{~A}$ ). In contrast, the isoenzyme CYP26B1 showed little to no changes in mRNA levels (Supplemental Figure 3A). Conditioned media derived from MM cells also upregulated CYP26A1 in BM stromal cells, although to a lesser extent (Supplemental Figure 3B). This could be explained by the presence of physical interactions in coculture experiments, or the lack of continuous production of soluble ligands by MM cells in conditioned media experiments. Consistent with the latter, stromal CYP26A1 was highly upregulated when MM and BM stromal cells were separated by a Transwell that prevented physical contact but allowed the diffusion of soluble factors (Figure 3A).

MM cells produce a variety of soluble factors including cytokines (IL-1, IL-3, IL-6, TNF- $\alpha$ ) as well as Hedgehog ligands such as sonic hedgehog (SHH) $(25,26)$, which could impact the BM stromal compartment. Therefore, we next investigated whether any of these factors was responsible for the observed upregulation of CYP26A1 on BM stromal cells. Of the soluble factors tested, only SHH produced a sustained overexpression of CYP26A1, while IL-1, IL-3, IL-6, and TNF- $\alpha$ had no significant effects (Figure 3B). Whereas $\mathrm{SHH}$ is expressed by BM stromal cells and thus may be able to activate the Hedgehog pathway in an autocrine manner (27), its expression was considerably higher in MM cells (Figure 3C) compared with that detected in BM stroma, suggesting that paracrine activation may play a dominant role. Consistent with this, we observed a statistically significant correlation between the mRNA levels of SHH in MM cells and activation of stromal Hedgehog signaling as determined by protein patched homolog 1 (PTCH1) expression (Figure 3D). Moreover, activation of stromal Hedgehog significantly correlated with CYP26A1 upregulation (Supplemental Figure 3C). Specifically, MM1S cells with the highest expression of SHH also induced the highest expression of both PTCH1 and CY26A1 in stromal cells (Figure 3D and Supplemental Figure 3C). SHH has a half-life of less than 1 hour, which may explain the reduced effect of MM-conditioned media on stromal CYP26A1 expression compared with that observed in coculture and Transwell experiments (28).

To confirm the role of paracrine Hedgehog on this interaction, smoothened (Smo), a membrane receptor that transduces SHH sig- 

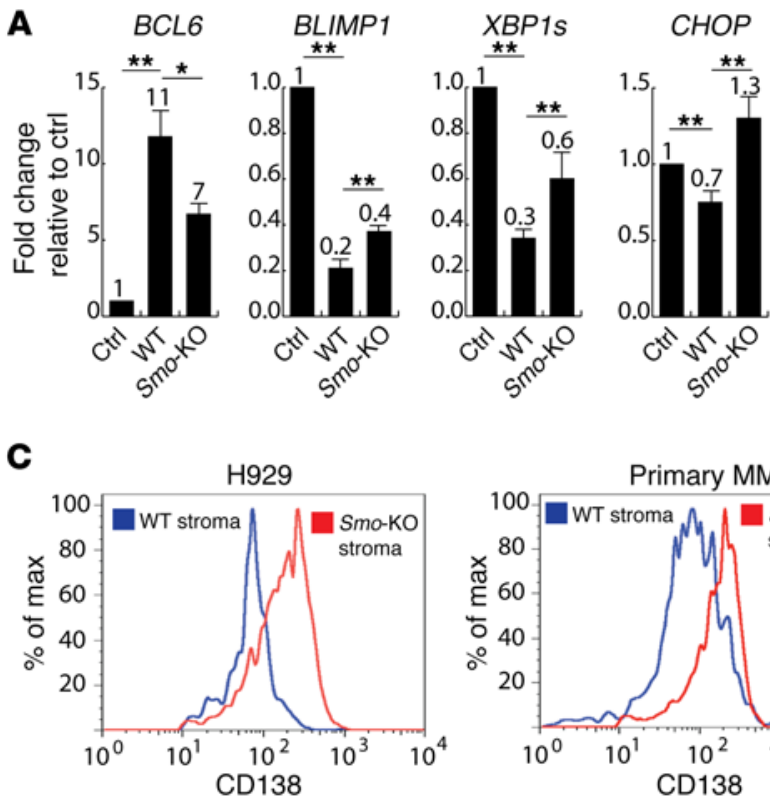

$\mathbf{E}$
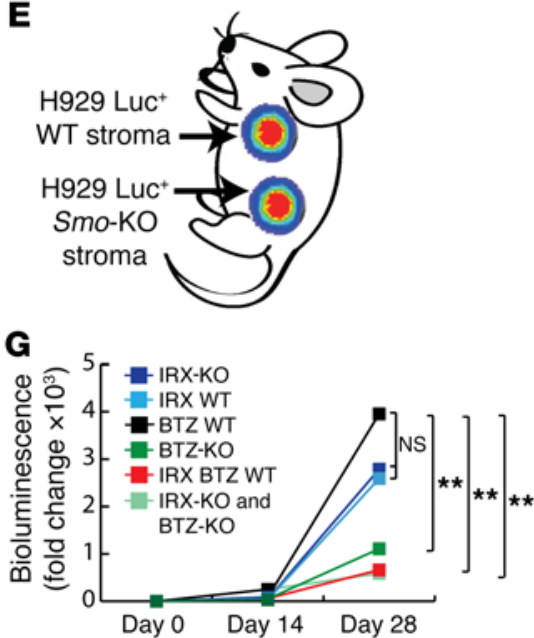
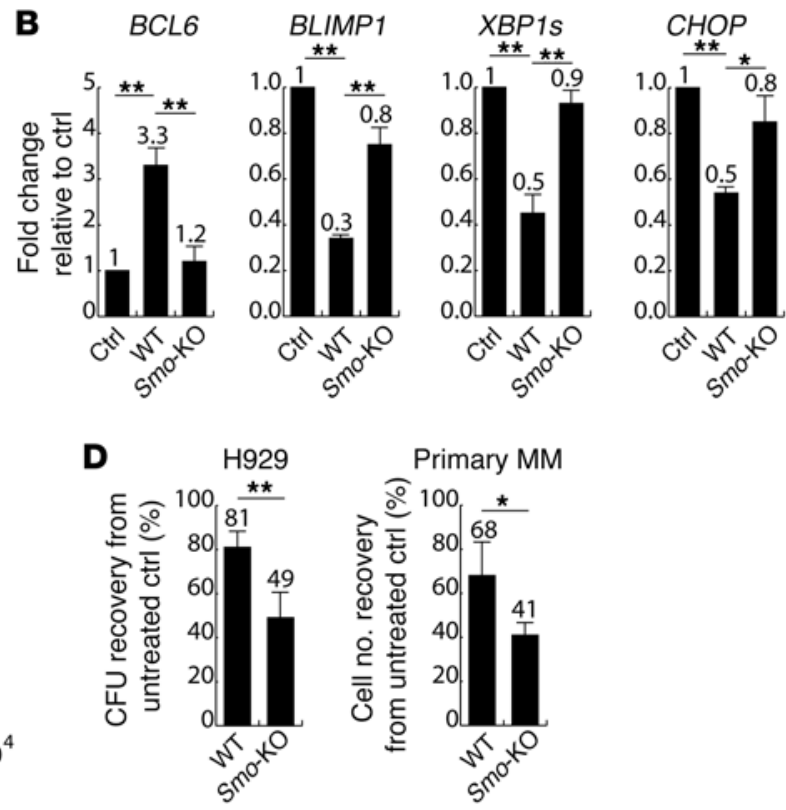

Primary MM

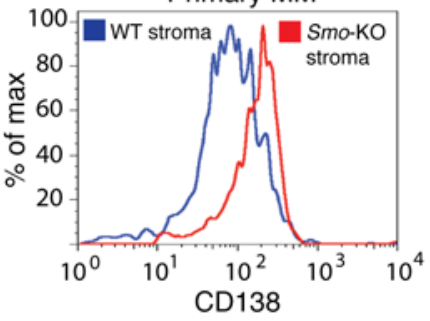

$\mathbf{F}$

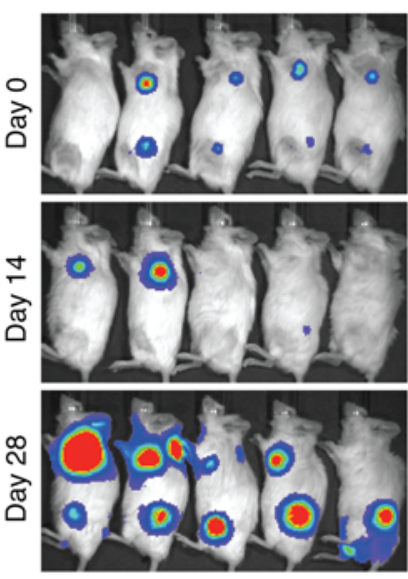

BTZ

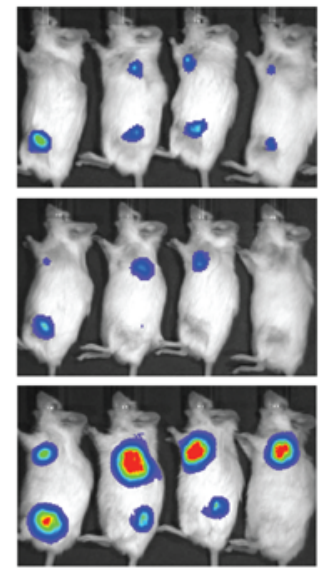

$\mathrm{IRX}+\mathrm{BTZ}$

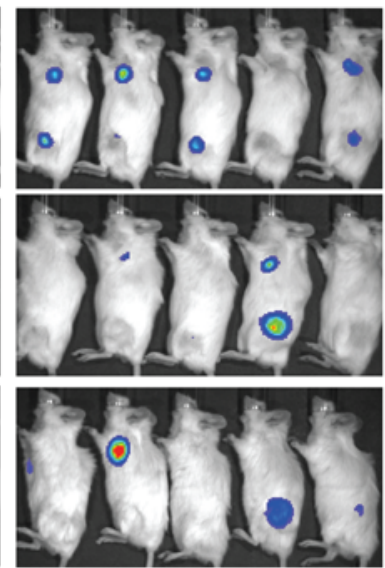

Figure 4. Effects of paracrine SHH signaling on plasma cell differentiation and BTZ sensitivity of MM cells. (A and B) Relative quantification of $B C L 6$ (B cell marker), BLIMP, XBP1s, and CHOP (PC markers) in $\mathrm{H} 929$ cells (A) and primary CD138 ${ }^{+}$MM cells (B) from 3 different patient samples incubated for 5 days either in the absence of stroma (Ctrl) or cocultured with WT or Smo-KO stromal cells. Expression in untreated liquid conditions was set at 1. (A and B) Data represent the mean $\pm \mathrm{SEM}$. ${ }^{*} P \leq 0.05$ and ${ }^{* *} P \leq 0.01$, by repeated-measures 1 -way ANOVA to determine statistical significance between treatment groups; $P$ values were corrected for multiple comparisons using Dunnett's test. (C) Flow cytometric analysis of CD138 in H929 or primary CD138 ${ }^{+}$MM cells cocultured for 5 days with WT or Smo-KO stromal cells. Data are representative of 3 independent experiments. (D) BTZ sensitivity of H929 (CFU recovery) or primary CD138 ${ }^{+} \mathrm{MM}$ cells (cell number recovery) from 3 different patient samples. MM cells were treated with BTZ (2.5 nM) for 48 hours after coculture with WT or Smo-KO stromal cells for 5 days. CFU or cellular recovery was normalized to each condition in the absence of BTZ. Data represent the mean \pm SEM. ${ }^{*} P \leq 0.05$ and ${ }^{* *} P \leq 0.01$, by 2-tailed Student's $t$ test. (E) Cartoon depicting the experimental design of subcutaneous xenografts. Each mouse carried 2 tumors consisting of $\mathrm{H929}$ Luc $^{+}$cells and either WT or Smo-KO stroma. (F) Bioluminescent images of xenografts upon treatment with IRX ( $n=10$ ), BTZ $(n=9)$, or a combination of both $(n=10)$ for 4 weeks. (C) Fold change in bioluminescence (photons/second) from day 0 of treatment. Results are the average of 9 to 10 xenografts per treatment group. ${ }^{*} P \leq 0.05$ and ${ }^{* *} P \leq 0.01$, by unpaired, 2 -tailed Student's $t$ test.

naling, was knocked out at the genomic level in the mesenchymal compartment. For this, BM mesenchymal cells derived from $S m o^{f / f l}$ mice were transduced with a retroviral vector encoding Cre recombinase (Smo-KO stroma). Mouse $S m o^{t / f l}$ stromal cells transduced with an empty retroviral vector were used as a control (WT stroma). The transduced BM stromal cells were cocultured with MM cells for 24 hours. As expected, Smo-KO stroma had a decreased ability to upregulate Cyp26a1 in response to MM cells compared with WT stroma (Figure 3E). Similarly, the SMO inhibitor cyclopamine partially overcame stromal Cyp26a1 upregulation by MM cells (Supplemental Figure 3D). These data suggest that MM cells modulate stromal CYP26 expression at least in part via paracrine $\mathrm{SHH}$.

Paracrine Hedgehog produced by MM cells reinforces a protective microenvironment. Given our observations that stromal CYP26 activity may be responsible for BTZ resistance in MM cells, we assessed whether paracrine Hedgehog secreted by MM cells rein- 
forces a chemoprotective niche by regulating retinoid metabolism. We first investigated whether modulation of Hedgehog signaling paralleled the retinoid-dependent phenotypes observed previously. Disruption of paracrine Hedgehog signaling in Smo-KO stroma cocultures partially restored PC differentiation (downregulation of BCL6 and upregulation of BLIMP1, XBP1, and CHOP) in $\mathrm{H} 929$ (Figure $4 \mathrm{~A}$ ) and primary CD138 ${ }^{+} \mathrm{MM}$ cells (Figure 4B). Surface expression of CD138 (Figure 4C) was also restored in the presence of $\mathrm{Smo-KO}$ stroma. As expected, these findings were associated with an increased sensitivity to BTZ of MM cells treated in the presence of Smo-KO stroma compared with WT stroma (Figure 4D).

To demonstrate that paracrine Hedgehog indeed induces a BTZ-resistant phenotype by increasing the ability of BM stroma to inactivate retinoids, we rescued Cyp26a1 expression in Smo-KO stroma via lentivirus-mediated gene transfer (pBABE-Cyp26a1) in order to achieve comparable CYP26A1 levels in WT (WT-Cyp26a1) and Smo-KO (Smo-KO-Cyp26a1) stromal cells (Supplemental Figure 4A). If the role of paracrine Hedgehog was independent of retinoid signaling, the relative inability of $\mathrm{Smo}-\mathrm{KO}$ stroma to induce a B cell phenotype and BTZ resistance should have persisted even after Cyp26a1 upregulation. We found, however, that Cyp26a1 overexpression rescued the ability of Smo-KO stroma to induce a $B$ cell phenotype and restored the expression of differentiation markers (Supplemental Figure 4B) and BTZ resistance (Supplemental Figure $4 \mathrm{C}$ ) to levels comparable to those detected in WT and WT-Cyp26a1 stroma coculture conditions. This finding is consistent with the hypothesis that paracrine Hedgehog reinforces a protective niche via Cyp26a1 upregulation.

To study to what extent an RA-low environment created by the $\mathrm{BM}$ stroma and enhanced by MM cells via paracrine Hedgehog signaling contributes to BTZ resistance, we constructed a xenograft model of MM-niche interactions. Each mouse carried 2 subcutaneous tumors consisting of H929 Luc ${ }^{+}$cells and either WT (anterior tumors) or Smo-KO stroma (posterior tumors) (Figure $4 \mathrm{E}$ ). Mice were treated with IRX (10 mg/kg i.p. daily), BTZ $(0.5 \mathrm{mg} /$ $\mathrm{kg}$ i.p. twice weekly), or a combination of both. We found that the growth of tumors bearing WT or Smo-KO stroma was not different in untreated or IRX-treated groups (Figure 4, F and G). Consistent with our in vitro data, tumors with WT stroma were refractory to BTZ treatment, as determined by an exponential increase in bioluminescence, while tumors carrying Smo-KO stroma showed a significant response (Figure 4G). Moreover, the combination of IRX and BTZ resulted in a significant and equivalent response, regardless of the phenotype of the stromal compartment (Figure 4G). While some tumors in the treatment group receiving combined IRX and BTZ appeared to have regressed completely, even after anatomical study, this was not the case for all the mice in this group. Flow cytometric analyses of the tumors after treatment revealed no differences in the in vivo growth of WT or Smo-KO stroma (data not shown). Taken together, these data suggest that paracrine Hedgehog secreted by MM cells modulates retinoid signaling and BTZ sensitivity in the BM niche via CYP26A1 upregulation.

\section{Discussion}

Given their high secretion of Ig, PCs are particularly sensitive to proteasome inhibition, and this accounts for the high remission rates achieved in MM patients treated with this family of drugs
(6). Nonetheless, BTZ has failed to achieve a cure. We previously showed that a population of MM cells, phenotypically similar to B cells, survive BTZ treatment and are able to differentiate into PCs and recapitulate the original disease (4). Despite efficient elimination of MM PCs, these MM B cells survive BTZ treatment and become the predominant cell population during MRD (5). Consequently, new therapeutic strategies targeting MM B cells are required. Here, we show that a retinoid-low microenvironment created by stromal CYP26 maintained an immature, BTZresistant phenotype in $\mathrm{MM}$. Thus, these data reveal a therapeutic opportunity to overcome BTZ resistance in the MM microenvironment using CYP26-resistant retinoids.

Despite being extensively studied in many hematological malignancies, the use of retinoids as differentiation therapy has proved beneficial only in patients with acute promyelocytic leukemia (APL) (29). Our previous studies suggest that CYP26 expression by BM stromal cells may explain the lack of a clinical benefit of natural retinoids, despite their in vitro activity (11). Recent studies have highlighted the efficacy of CYP-resistant synthetic retinoids in differentiating cancer cells and sensitizing them to targeted therapy. For instance, AM80 differentiates FMS-like tyrosine kinase 3/internal tandem duplication (FLT3/ITD) acute myeloid leukemia (AML) cells and increase their sensitivity to FLT3 inhibitors (30). Similarly, synthetic retinoids reverse a stem cell phenotype in BCR-ABL1 ${ }^{+}$leukemic lymphoblasts and substantially increase their responsiveness to tyrosine kinase inhibitor (TKI) therapy in vivo (31). Such strategies to bypass stromal CYP26 could expand the clinical effectiveness of retinoid therapy.

MM cells utilize physical contacts to maintain drug resistance and survive within the BM niche. Thus, therapeutic strategies to overcome stromal chemoprotection have focused on mobilization of malignant cells from the BM niche by targeting adhesion molecules or chemokines such as CXCR4 (18-20). Here, we show that $\mathrm{MM}$ cells exposed to a retinoid-low microenvironment acquire a BTZ-resistant phenotype that is maintained even after these cells are displaced from their niche. Initial clinical studies have shown improved response rates in relapse/refractory patients receiving the CXCR4 inhibitor plerixafor in combination with BTZ (20); however, our data suggest that such mobilization approaches may be insufficient to eliminate MM B cells.

Recent studies have demonstrated the existence of a bidirectional communication, in which not only stromal cells provide a chemoprotective niche, but also cancer cells actively shape and reinforce their microenvironment (21-24). The role of paracrine Hedgehog has been studied extensively in solid malignancies (32-35). In this system, ligands secreted by cancer cells activate the Hedgehog pathway in neighboring stromal cells, enhancing their chemoprotective properties via incompletely understood mechanisms. Our data suggest that paracrine Hedgehog may work at least in part by increasing the ability of stroma to inactivate retinoids through upregulation of CYP26 and thus to maintain a BTZ-resistant phenotype in MM. Interestingly, CYP26 upregulation is associated with an "activated stromal subtype" and a significantly worse prognosis in patients with pancreatic cancer (36), a disease in which paracrine Hedgehog signaling is well established. The extent to which Hedgehog ligands produced by cancer cells contribute to this "activated" stromal phenotype 
and high CYP26 levels is unknown. Moreover, BM mesenchymal cells migrate and become a relevant cell population in the stromal compartment of these tumors $(37,38)$. These studies raise the possibility that the stromal compartment in the $\mathrm{BM}$ and solid malignancies may respond to paracrine Hedgehog in a similar manner, namely that of CYP26 upregulation.

The endosteal region is the primary niche of MM (39), AML (40), and micrometastatic disease from solid tumors $(41,42)$. Within the osteoblastic region, these cancer cells maintain a quiescent, stem cell phenotype and are protected from chemotherapy-induced apoptosis. It is likely that these cancer cells rely on the same cues from the BM microenvironment as normal hematopoietic stem cells do to survive chemotherapy and perpetuate the disease. We previously showed that the BM microenvironment protected MM and AML cells by directly inactivating various chemotherapy agents via expression of CYP3A4 and other detoxifying enzymes. Here, we show another potential mechanism of microenvironment-mediated drug resistance: creation of a retinoid-low niche that maintains a drug-resistant B cell phenotype. Our finding that a CYP26-resistant retinoid potentiated the activity of BTZ against MM in the BM niche may provide a therapeutic opportunity to bypass this mechanism of resistance. Since retinoids induce cell differentiation in various malignancies (43-45), it is plausible that stromal CYP26 activity creates retinoid-low niches and provides cancer cells in general with a mechanism of chemoresistance.

\section{Methods}

Cell cultures. All cell lines were purchased from the American Type Culture Collection. H929, MM1s, and U266 cells were cultured in RPMI 1640 (Gibco, Thermo Fisher Scientific) with 10\% FCS (SigmaAldrich), $2 \mathrm{mM}$ L-glutamine (Life Technologies, Thermo Fisher Scientific), and $100 \mu \mathrm{g} / \mathrm{ml}$ penicillin-streptomycin (P/S) (Gibco, Thermo Fisher Scientific). OP-9 cells were cultured in $\alpha$-MEM (Gibco, Thermo Fisher Scientific), 20\% FCS, L-glutamine, and P/S. Cell lines were authenticated by short-tandem repeat profiling.

Primary MM cells were obtained from patients with newly diagnosed or relapsed MM under an IRB-approved protocol at Johns Hopkins. Briefly, mononuclear cells were isolated from fresh BM aspirates by density gradient centrifugation (Ficoll-Paque; GE Healthcare Life Sciences); CD138 ${ }^{+}$cells were then selected via magnetic beads and columns according to the manufacturer's instructions (Miltenyi Biotec) and incubated in RPMI 1640, 10\% FCS, L-glutamine, and $\mathrm{P} / \mathrm{S}$ at $37^{\circ} \mathrm{C}$.

Primary human BM stromal cells were derived from aspirates collected from healthy donors under an IRB-approved protocol at Johns Hopkins, as we previously described (1). Briefly, total mononuclear cells isolated from BM aspirates were cultured in Iscove's modified Dulbecco's medium (IMDM) (Sigma-Aldrich) supplemented with $10 \%$ horse serum (Sigma-Aldrich), 10\% FCS, $10^{-5} \mathrm{M}$ hydrocortisone 21-hemisuccinate (Sigma-Aldrich), P/S, and $0.1 \mathrm{mM} \beta$-mercaptoethanol ( $\beta$-ME) (FBMD1 media) (46). The following day, cells in suspension were removed by washing twice with PBS (Gibco, Thermo Fisher Scientific), and the media were replaced. Attached stromal cells were incubated at $33^{\circ} \mathrm{C}$ until a confluent monolayer was obtained. Mouse primary $\mathrm{BM}$ stromal cells were isolated following the same protocol, after isolation of total BM mononuclear cells from mouse femurs.

Vectors and viral supernatants. To generate Smo-KO and WT stroma, BM stromal cells were derived from $S m o^{f / f l}$ mice and trans- duced with the retroviral vector PIG-Cre encoding Cre-recombinase (Addgene; catalog 50935) (47) or a control vector (Addgene; catalog 18751), respectively. Successfully infected cells were selected using $4 \mu \mathrm{g} / \mathrm{ml}$ puromycin (Sigma-Aldrich) for 5 days and confirmed by expression of GFP via flow cytometry. The pLenti-CMV-LUC-Puro lentiviral vector (plasmid 17477) was used to generate $\mathrm{H} 929 \mathrm{Luc}^{+}$cells as previously described (48).

To generate CYP26A1-overexpressing stromal cells, WT and Smo$\mathrm{KO}$ stromal cells were transduced with the lentiviral vector pBABEneo (Addgene; catalog 1767) (49) that had been engineered to encode CYP26A1. Briefly, Cyp26a1 cDNA (Origene) was amplified via PCR using primers incorporating the restriction sites BamHI and EcoRI and cloned into the pCR2.1 vector. Cyp26a1 cDNA was confirmed via Sanger sequencing, and the fragment was isolated after digestion with the restriction enzymes BamHI and EcoRI and subcloned into the corresponding sites of the $\mathrm{pBABE}$ vector. Lentiviral particles were produced as previously described (1). Successfully infected stromal cells were selected using $3 \mu \mathrm{g} / \mathrm{ml} \mathrm{G}$-418 (Sigma-Aldrich) for 10 days, and expression of Cyp26a1 was confirmed by qRT-PCR.

Coculture experiments. Coculture experiments were performed as previously described (1). Briefly, 24-well plates (Thermo Fisher Scientific) were coated with $0.1 \%$ gelatin (Sigma-Aldrich) in PBS for 30 minutes at $37^{\circ} \mathrm{C}$. The gelatin solution was removed, and the stromal cells were cultured overnight at a density of $5 \times 10^{4}$ cells/well to obtain a confluent monolayer. At that time, MM cell lines or primary MM cells $\left(1 \times 10^{5}\right.$ in $\left.2 \mathrm{ml}\right)$ were added to the stroma cultures. The stroma cocultures were incubated at $37^{\circ} \mathrm{C}$ in RPMI containing $10 \%$ FCS, L-glutamine, and P/S, with or without AGN ( $1 \mu \mathrm{M}$ for 5 days), R115866 $(1 \mu \mathrm{M}$ for 5 days), IRX ( $1 \mu \mathrm{M}$ for 5 days), or BTZ (2.5 nM for 48 hours).

Transwell experiments. For Transwell experiments, 6-well plates (Thermo Fisher Scientific) were coated with $0.1 \%$ gelatin (SigmaAldrich) in PBS for 30 minutes at $37^{\circ} \mathrm{C}$. The gelatin solution was removed, and the stromal cells were cultured overnight in FBMD1 media at a density of $10 \times 10^{4}$ cells/well in $2 \mathrm{ml}$ of media to obtain a confluent monolayer. At that time, Transwell inserts (Corning) were placed over the stroma cultures, and MM cell lines $\left(1 \times 10^{6} \mathrm{in} 1 \mathrm{ml}\right)$ were seeded in the Transwell for 24 hours at $37^{\circ} \mathrm{C}$. Following this incubation, Transwell and MM cells were removed, and stromal cells were detached from the wells and analyzed by qRT-PCR for CYP26 expression.

Mobilization experiments. MM cells were separated from BM stromal cells by gently pipetting several times around the well. Detached cells were centrifuged, resuspended in fresh media, and incubated in a 24-well plate for 1 hour at $37^{\circ} \mathrm{C}$. During this short incubation period, contaminating stromal cells attached to the well, while MM cells remained in suspension. MM cells were then recovered by gently pipetting. This protocol was used for qRT-PCR and CFU coculture experiments. The purity achieved using this protocol was confirmed by flow cytometry to be $98 \%-99 \%$ MM cells and less than 2\% contaminating stroma.

Clonogenic assays. Clonogenic assays were performed as previously described (1). Briefly, after treatment, MM cells were collected, washed with PBS, and plated at a density of 5,000 cells $/ \mathrm{ml}$ in $1 \mathrm{ml}$ of $1.32 \%$ methylcellulose (Sigma-Aldrich) supplemented with 30\% FBS, $10 \%$ BSA (Sigma-Aldrich), L-glutamine, P/S, and $0.1 \mathrm{mM} \beta$-ME. Cells were plated in triplicate in $35-\mathrm{mm}$ culture dishes, incubated at $37^{\circ} \mathrm{C}$, and scored for the presence of colonies 14 days later.

qRT-PCR. qPCR assays were performed as we have described previously (1). Briefly, total RNA was extracted using the RNeasy Mini 
Kit (QIAGEN) according to the manufacturer's instructions. cDNA was synthesized by reverse transcription using the iScript cDNA Synthesis Kit (Bio-Rad). qRT-PCR was performed with iTaq SYBR Green Supermix (Bio-Rad) using sequence specific primers. Gene expression was normalized to $G A P D H$, and relative quantification was calculated using $\Delta \Delta \mathrm{Ct}$. All experiments were performed in duplicate and run on the Bio-Rad CFX96 machine.

Flow cytometry. Flow cytometric experiments were performed as previously described (10). Following treatment, MM cells were collected, washed with PBS, and stained for 15 minutes at room temperature with phycoerythrin-conjugated (PE-conjugated) anti-CD138 (BD Biosciences; catalog 347206). Cells were washed to remove unbound antibody and evaluated in a FACSCalibur system (BD Biosciences). Stromal cells were identified by GFP expression, and viable cells were identified using 7-aminoactinomycin D (7-AAD) (Invitrogen, Thermo Fisher Scientific). To calculate cell numbers, alive GFP- cells were normalized to calibration beads (BD Biosciences).

Mouse xenografts. Subcutaneous xenografts were generated as previously described (1). Briefly, $1 \times 10^{6} \mathrm{H} 929 \mathrm{Luc}^{+}$cells and $1 \times 10^{6}$ mouse $\mathrm{BM}$ stromal cells were resuspended in $100 \mu \mathrm{l}$ Matrigel (Invitrogen, Thermo Fisher Scientific) diluted with RPMI (1:1) and injected subcutaneously into 16-week-old male NSG mice. After 4 days, treatment with BTZ (0.5 mg/kg i.p. twice weekly) and IRX (10 mg/kg i.p. daily) was initiated. Tumor burden was assessed by bioluminescence using the In Vivo Imaging System (PerkinElmer). For imaging, mice were exposed to $120 \mathrm{mg} / \mathrm{kg}$ D-luciferin (PerkinElmer) via intraperitoneal injection 10-5 minutes before imaging and were anesthetized using isoflurane (VetOne). Images were analyzed with Living Image Software, version 2.5 (PerkinElmer), and data were quantified as photons/second.

For the systemic MM model, $2 \times 10^{6} \mathrm{Luc}^{+} / \mathrm{GFP}^{+} \mathrm{H} 929$ cells were injected via the tail vein into 16 -week-old male NSG mice. After engraftment, as determined by an exponential increase in bioluminescence, mice were treated with BTZ $(0.5 \mathrm{mg} / \mathrm{kg}$ i.p.) twice weekly and with IRX (10 mg/kg) once daily. Tumor burden was assessed by bioluminescence, as above.

Statistics. We first evaluated whether the treatment groups were different from the controls using 1-way ANOVA. If the ANOVA test yielded a statistically significant result, then the difference between the control group and each treatment group was evaluated, with the $P$ values adjusted for multiple comparisons using Dunnett's test. For experiments in which only 2 sets of data were analyzed, statistical significance was evaluated using an unpaired, 2-tailed Student's $t$ test. Pearson's $R$ value for correlation and $P$ values were calculated using GraphPad Prism 7(GraphPad Software).

Study approval. All animal studies were performed under a protocol approved by the Johns Hopkins Animal Care and Use Committee and complied with NIH guidelines (Guide for the Care and Use of Laboratory Animals. National Academies Press. 2011).

\section{Author contributions}

SA, WHM, RJJ, and GG designed the research studies. SA, DH, YC, and $\mathrm{MM}$ conducted the experiments and acquired the data. SA, RJJ, and GG analyzed the data. SA, RJJ, and GG wrote the manuscript. CDG contributed reagents and materials and reviewed the manuscript. SA, RV, and GG performed the statistical analyses.

\section{Acknowledgments}

This work was supported by grants from the National Heart, Lung, and Blood Institute (1K08 HL127269-01, to GG); the Commonwealth Foundation Pilot Project (to GG); the Leukemia and Lymphoma Society (14086277, to RJJ and GG); the National Cancer Institute (P30 CA006973, to RJJ); and the NIH (P01 CA015396, to RJJ).

Address correspondence to: Gabriel Ghiaur, 1650 Orleans St., Room 243, Baltimore, Maryland 21231, USA. Phone: 410.502.3183; E-mail: gghiaur1@jhmi.edu.
1. Alonso S, et al. Human bone marrow niche chemoprotection mediated by cytochrome $\mathrm{P} 450$ enzymes. Oncotarget. 2015;6(17):14905-14912.

2. Hideshima T, Mitsiades C, Tonon G, Richardson PG, Anderson KC. Understanding multiple myeloma pathogenesis in the bone marrow to identify new therapeutic targets. Nat Rev Cancer. 2007;7(8):585-598.

3. Roccaro AM, et al. BM mesenchymal stromal cellderived exosomes facilitate multiple myeloma progression. J Clin Invest. 2013;123(4):1542-1555.

4. Matsui W, et al. Clonogenic multiple myeloma progenitors, stem cell properties, and drug resistance. Cancer Res. 2008;68(1):190-197.

5. Leung-Hagesteijn C, et al. Xbp1s-negative tumor $B$ cells and pre-plasmablasts mediate therapeutic proteasome inhibitor resistance in multiple myeloma. Cancer Cell. 2013;24(3):289-304.

6. Obeng EA, Carlson LM, Gutman DM, Harrington WJ Jr, Lee KP, Boise LH. Proteasome inhibitors induce a terminal unfolded protein response in multiple myeloma cells. Blood. 2006;107(12):4907-4916.

7. Walter P, Ron D. The unfolded protein response: from stress pathway to homeostatic regulation. Science. 2011;334(6059):1081-1086.
8. Yaccoby S. The phenotypic plasticity of myeloma plasma cells as expressed by dedifferentiation into an immature, resilient, and apoptosis-resistant phenotype. Clin Cancer Res. 2005;11(21):7599-7606.

9. Dezorella N, et al. Mesenchymal stromal cells revert multiple myeloma cells to less differentiated phenotype by the combined activities of adhesive interactions and interleukin-6. Exp Cell Res. 2009;315(11):1904-1913.

10. Ghiaur G, Yegnasubramanian S, Perkins B, Gucwa JL, Gerber JM, Jones RJ. Regulation of human hematopoietic stem cell self-renewal by the microenvironment's control of retinoic acid signaling. Proc Natl Acad Sci U S A. 2013;110(40):16121-16126.

11. Su M, et al. All-trans retinoic acid activity in acute myeloid leukemia: role of cytochrome P450 enzyme expression by the microenvironment. PLoS One. 2015;10(6):e0127790.

12. Ertesvag A, Aasheim HC, Naderi S, Blomhoff HK. Vitamin A potentiates CpG-mediated memory B-cell proliferation and differentiation: involvement of early activation of p38MAPK. Blood. 2007;109(9):3865-3872.

13. Mora JR, et al. Generation of gut-homing IgA- secreting B cells by intestinal dendritic cells. Science. 2006;314(5802):1157-1160.

14. Nutt SL, Hodgkin PD, Tarlinton DM, Corcoran LM. The generation of antibody-secreting plasma cells. Nat Rev Immunol. 2015;15(3):160-171.

15. Shaffer AL, et al. XBP1, downstream of Blimp-1, expands the secretory apparatus and other organelles, and increases protein synthesis in plasma cell differentiation. Immunity. 2004;21(1):81-93.

16. Niu H, Chacko J, Hadwiger G, Welch JS. Absence of natural intracellular retinoids in mouse bone marrow cells and implications for PML-RARA transformation. Blood Cancer J. 2015;5:e284.

17. Chee LC, Hendy J, Purton LE, McArthur GA. ATRA and the specific RAR $\alpha$ agonist, NRX195183, have opposing effects on the clonogenicity of pre-leukemic murine AML1-ETO bone marrow cells. Leukemia. 2013;27(6):1369-1380.

18. Azab AK, et al. CXCR4 inhibitor AMD3100 disrupts the interaction of multiple myeloma cells with the bone marrow microenvironment and enhances their sensitivity to therapy. Blood. 2009;113(18):4341-4351.

19. Passaro D, et al. CXCR4 is required for leukemiainitiating cell activity in T cell acute lymphoblastic leukemia. Cancer Cell. 2015;27(6):769-779. 
20. Ghobrial IM, et al. Phase I/II trial of plerixafor and bortezomib as a chemosensitization strategy in relapsed or relapsed/refractory multiple myeloma. Blood. 2014;124(21):5777.

21. Schepers K, et al. Myeloproliferative neoplasia remodels the endosteal bone marrow niche into a self-reinforcing leukemic niche. Cell Stem Cell. 2013;13(3):285-299.

22. Medyouf H, et al. Myelodysplastic cells in patients reprogram mesenchymal stromal cells to establish a transplantable stem cell niche disease unit. Cell Stem Cell. 2014;14(6):824-837.

23. Paggetti J, et al. Exosomes released by chronic lymphocytic leukemia cells induce the transition of stromal cells into cancer-associated fibroblasts. Blood. 2015;126(9):1106-1117.

24. Ghiaur G, Wroblewski M, Loges S. Acute myelogenous leukemia and its microenvironment: a molecular conversation. Semin Hematol. 2015;52(3):200-206.

25. Edwards CM, Zhuang J, Mundy GR. The pathogenesis of the bone disease of multiple myeloma. Bone. 2008;42(6):1007-1013.

26. Liu Z, et al. A critical role of autocrine sonic hedgehog signaling in human $\mathrm{CD} 138^{+}$myeloma cell survival and drug resistance. Blood. 2014;124(13):2061-2071.

27. Dierks C, et al. Essential role of stromally induced hedgehog signaling in B-cell malignancies. Nat Med. 2007;13(8):944-951.

28. Guy RK. Inhibition of sonic hedgehog autoprocessing in cultured mammalian cells by sterol deprivation. Proc Natl Acad Sci U S A. 2000;97(13):7307-7312.

29. Huang ME, et al. Use of all-trans retinoic acid in the treatment of acute promyelocytic leukemia. Blood.1988;72(2):567-572.
30. Ma HS, et al. FLT3 inhibition and retinoid signaling overcome stromal protection to target FLT3/ITD-expressing leukemia stem cells in the bone marrow microenvironment. Blood. 2015;126(23):790-790.

31. Churchman ML, et al. Efficacy of retinoids in IKZF1-mutated BCR-ABL1 acute lymphoblastic leukemia. Cancer Cell. 2015;28(3):343-356.

32. Yauch RL, et al. A paracrine requirement for hedgehog signalling in cancer. Nature. 2008;455(7211):406-410.

33. Olive KP, et al. Inhibition of Hedgehog signaling enhances delivery of chemotherapy in a mouse model of pancreatic cancer. Science. 2009;324(5933):1457-1461.

34. Bailey JM, et al. Sonic hedgehog promotes desmoplasia in pancreatic cancer. Clin Cancer Res. 2008;14(19):5995-6004.

35. Collins MA, et al. Oncogenic Kras is required for both the initiation and maintenance of pancreatic cancer in mice. J Clin Invest. 2012;122(2):639-653.

36. Moffitt RA, et al. Virtual microdissection identifies distinct tumor- and stroma-specific subtypes of pancreatic ductal adenocarcinoma. Nat Genet. 2015;47(10):1168-1178.

37. Direkze NC, et al. Bone marrow contribution to tumor-associated myofibroblasts and fibroblasts. Cancer Res. 2004;64(23):8492-8495.

38. Quante M, et al. Bone marrow-derived myofibroblasts contribute to the mesenchymal stem cell niche and promote tumor growth. Cancer Cell. 2011;19(2):257-272.

39. Chen Z, Orlowski RZ, Wang M, Kwak L, McCarty N. Osteoblastic niche supports the growth of quiescent multiple myeloma cells. Blood. 2014;123(14):2204-2208.

40. Ishikawa F, et al. Chemotherapy-resistant human
AML stem cells home to and engraft within the bone-marrow endosteal region. Nat Biotechnol. 2007;25(11):1315-1321.

41. Weilbaecher KN, Guise TA, McCauley LK. Cancer to bone: a fatal attraction. Nat Rev Cancer. 2011;11(6):411-425.

42. Shiozawa Y, et al. Human prostate cancer metastases target the hematopoietic stem cell niche to establish footholds in mouse bone marrow. J Clin Invest. 2011;121(4):1298-1312.

43. Ginestier C, et al. Retinoid signaling regulates breast cancer stem cell differentiation. Cell Cycle. 2009;8(20):3297-3302.

44. Rosewicz S, et al. Retinoids: effects on growth, differentiation, and nuclear receptor expression in human pancreatic carcinoma cell lines. Gastroenterology. 1995;109(5):1646-1660.

45. Kelly WK, et al. The development of biologic end points in patients treated with differentiation agents: an experience of retinoids in prostate cancer. Clin Cancer Res. 2000;6(3):838-846.

46. Breems DA, Blokland EA, Neben S, Ploemacher RE. Frequency analysis of human primitive haematopoietic stem cell subsets using a cobblestone area forming cell assay. Leukemia. 1994;8(7):1095-1104.

47. Chen Z, et al. Crucial role of p53-dependent cellular senescence in suppression of Pten-deficient tumorigenesis. Nature. 2005;436(7051):725-730.

48. Campeau E, et al. A versatile viral system for expression and depletion of proteins in mammalian cells. PLoS One. 2009;4(8):e6529.

49. Morgenstern JP, Land H. Advanced mammalian gene transfer: high titre retroviral vectors with multiple drug selection markers and a complementary helper-free packaging cell line. Nucleic Acids Res. 1990;18(12):3587-3596. 